http://dx.doi.org/10.12795/PH.2003.v17.i02.06

\title{
¿UN TEATRO POLÍTICO RADICAL? LA MIRADA CONSCIENTE DE CARYL CHURCHILL Y CHARLOTTE KEATLEY
}

\author{
Carmen García Navarro \\ Universidad de Sevilla \\ Grupo de Investigación Escritoras y Escrituras
}

\begin{abstract}
This article deals with a selection of works belonging to the literary production of two British playwrights, Caryl Churchill and Charlotte Keatley, who make evident their concern with the different ways of representing the female discourse and its status as a cultural construction. Both authors will be considered within the context of their age, by paying attention to their skills, methods and technical devices when dealing with questions of gender studies and gender discourse.
\end{abstract}

El hecho de que Caryl Churchill (Londres, 1938) o Charlotte Keatley (Londres, 1960), perteneciente a una generación posterior, sean dramaturgas de éxito, así como innovadoras tanto en la técnica como en la forma, es alentador y a la vez significativo para el teatro británico.

La consideración del teatro como tema de estudio plantea la necesidad de pensar en este género como en una manifestación artística que va dirigida a un público presente en el momento de la representación de la obra. Por ello, y teniendo en cuenta la nómina de autoras británicas que expresan su preocupación por los modos de representación del discurso femenino, hay que explicar, antes que nada, las líneas maestras del trabajo de aquéllas que se tratarán aquí, sin obviar la consideración de otras que comparten la visión del mundo que se expone en las obras que se comentarán. Así, es obvio que el hecho de compartir la experiencia de una coyuntura histórica determinada provocará el uso de un lenguaje escénico que acompaña a distintos dramaturgos y dramaturgas, si bien puede suceder que éstos se hallen muchas veces inmersos en distintas escuelas o tendencias que coexisten a lo largo de una o más generaciones.

Un aspecto que caracteriza la producción de Churchill y de Keatley, aunque en este caso cabría citar también otros nombres, como Timberlake Wertenbaker, por ejemplo, consiste en que algunas de sus obras han sido elaboradas a través de una extensa labor de colaboración con asistentes a talleres de escritura teatral. Se hablaría, en este caso, de factores que integran una tendencia teatral dentro de un período, compartida por distintos autores o autoras. Tanto Churchill como Keatley se han involucrado en esta tarea desde los inicios de sus respectivas trayectorias, siendo así que algunas de sus piezas han sido llevadas a cabo mediante su participación con actores, directores y alumnos asistentes a cursos de escritura teatral, todo ello en etapas que se corresponden con la creación como en la producción y realización de dichas obras. Esta característica, que define gran parte de su pauta de trabajo y que influye en el modo de concebir la estructura y la textura de sus obras 
es más evidente, si cabe, en el caso de Churchill porque empezó a escribir a principios de los años 60 y cuenta en su haber con más de una treintena de piezas dramáticas, sin mencionar sus adaptaciones y traducciones de obras de otros autores. Existe la posibilidad de disfrutar de una perspectiva que, si bien no es muy extensa en el tiempo, permite valorar la evolución de Churchill como autora teatral.

Además, el hecho de haber trabajado en colaboración con personas de diferentes grupos de teatro ha llevado a Churchill y a Keatley a concentrar su atención en los matices del ritmo, el habla y el acento. Estos elementos ejercen una función primordial en la creación de los personajes, así como en la forma de entender el tono o el propósito de sus obras y juegan un papel decisivo a la hora de iluminar los aspectos más sobresalientes de sus creaciones.

Si se piensa en el tipo de teatro necesario en nuestros días y, por lo tanto, en una declaración de intenciones sobre la que se pueda empezar este estudio, encuentro que será útil afirmar que necesitamos un tipo de teatro que muestre y libere el conjunto de tendencias propio de un período histórico determinado de las relaciones humanas. En él se desarrollaría la acción pero, sobre todo, en él se haría uso de las ideas y de las emociones que contribuyen a transformar ese teatro. Por lo que se refiere al caso concreto de Churchill y Keatley, lo anterior se explica cuando se comprueba la realidad de su compromiso, en grados de diferente alcance, con varios aspectos políticos y sociales de la realidad que les ha tocado vivir, como por ejemplo las restricciones que sufre el individuo por causas de raza, género o clase, la presión del materialismo en las sociedades occidentales, o los efectos del colonialismo. Pero, al mismo tiempo, estas autoras en cierto modo subvierten las expectativas de los lectores en lo que se refiere a dichos temas, forzando así a la audiencia a prestar atención constantemente a las demandas y a los interrogantes de sus creaciones. Para ello, Churchill y Keatley utilizan distintas técnicas, como la ironía, o investigan sobre una gran diversidad de recursos escénicos, lo que motiva y provoca la toma de posición de la audiencia una y otra vez, facilitando a ésta la experiencia de enfrentarse con distintos e inesperados discursos.

Hay que señalar el período temporal en el que se enmarca la producción y posterior representación de sus obras. En los ejemplos de las obras de las que se ocupan estas páginas, he intentado proponer un material que ofrece una serie de características que ayuden a observar cómo el género ha tratado la realidad política, social y cultural de la época en la que ha sido escrito. A pesar de que la perspectiva temporal no es aún muy amplia (un período que apenas cubre las tres últimas décadas), sí resulta lo suficientemente esclarecedor el observar la producción teatral escrita en Gran Bretaña tras la década anterior a la de los años 80 y durante la primera mitad de los 90 . Ello es así porque estas autoras eran ya consagradas entonces o eran dramaturgas cuya producción se iba viendo cada vez más respetada por el público y la crítica. El estudio, o la aproximación al estudio de la producción teatral de la época mencionada debe tener en cuenta, por lo tanto, la coyuntura que vivía el panorama cultural británico de ese período y la coincidencia del mismo con los gobiernos de Margaret Thatcher y su sucesor John Major. Como la escena política se convierte en una referencia constante en los diversos procesos de la vida social, económica y cultural, el teatro será un ámbito en el que queden reflejadas las inquietudes de las autoras. Al igual que otras manifestaciones artísticas, el teatro experimentó una serie de vivencias que eran reflejo $\mathrm{y}$, en muchos casos, respuesta, al ambiente que reinaba en el 
conjunto de la sociedad británica. Hay que afirmar, por tanto, con Ángel Berenguer y Manuel Pérez que la actividad artística teatral es también un modo de revisión de la actualidad en la que ésta se inserta y que, por tanto, determina la manera en la que los autores y autoras expresan sus creaciones (Berenguer / Pérez 1998: 15). De hecho, hay que recordar la situación que vivían las artes y la cultura en el mencionado período en Gran Bretaña. Así, según Elaine Aston y Janelle Reinelt, los años del gobierno de Thatcher fueron especialmente difíciles, "especially those alternative, political or experimental companies and artists who did not fall under the wings of powerful institutions like the National Theatre or the RSC" (Aston / Reinelt, 2000: 14).

Puede decirse que los trabajos de estas dramaturgas están elaborados desde la reflexión más rigurosa y que provocan el debate. Asimismo, están pensadas como manifestaciones artísticas abiertas a los diferentes registros con los que se conjuga el arte dramático. Con sus propuestas, tanto Churchill como Keatley logran abordar los diversos discursos que se han articulado sobre las mujeres y sobre lo femenino como construcción cultural. En opinión de Keatley, se trata, en principio, de desmantelar los constructos de género que pertenecen al pasado y, al mismo tiempo, de hacer posible, mediante la palabra teatral, la existencia del saber propio (Keatley 1994: XXXIV). Ello pone de manifiesto el interés por construir obras en las que se investigue la representación, o las representaciones, de la voz femenina propia recurriendo al papel de la memoria y proyectando la tarea de reescribir el presente revisándolo de manera crítica. De este modo, los personajes femeninos de sus obras plantean las posibilidades de expresar cómo quieren vivir su futuro porque se les ofrece una vía para manifestar su rabia y su resentimiento, a pesar de que sea esa la manera de dar al traste con la imagen y el rol que se ha asociado tradicionalmente a la mujer buena (Keatley 1994: XXXIII).

Estas autoras crean personajes complejos que, en su mayoría, representan la ruptura de los estereotipos femeninos. Así, algunas de las cuestiones analizadas en las obras de Churchill, en concreto en Top Girls (1988) y The Skriker (1993), y de Keatley, en My Mother Said I Never Should (1988), fueron de variado signo. Dada la naturaleza de las obras en cuestión, esas interrogantes consistieron, fundamentalmente, en buscar cuál es el grado de igualdad, diferencia o subordinación que experimentan o al que se han visto sometidas sus protagonistas como sujetos sociales, como sujetos históricos, como ciudadanas, para conducirse del modo en que lo hacen en estas obras. De la misma manera, es interesante saber si las autoras habían logrado otorgar voz a quienes no la tienen o no la han tenido y si estas protagonistas femeninas, estas mujeres, eran reconocidas con sus cuerpos y con todo lo que ellos simbolizan. Siguiendo esta línea, era importante plantearse qué obstáculos tenían que sortear estas protagonistas y qué desórdenes habían causado en ellas las luchas que tendrían que afrontar para vencerlos. Por ejemplo, y concretando, qué grado de esfuerzo supone el enfrentarse a la idea de la maternidad, a la posibilidad de vivir una vida propia que no asuma el deber de perpetuar los modos de pensamiento y de actuación de las generaciones pasadas, a la influencia materna, y qué consecuencias se derivan de ello para las mujeres que han tomado conciencia de la necesidad implícita de una toma de posición. Derivada de la toma de posición, o implícita en ella, la conciencia de la necesidad de la ciudadanía, de ser ciudadanas con derechos, oportunidades y obligaciones, en el seno de las sociedades democráticas occidentales, en este caso en la sociedad británica posterior a la Segunda Guerra Mundial. Al hilo de lo anterior, viene al 
caso atender a la rotunda afirmación de Keatley cuando habla de su propia obra "as a violent one, and [in] the way that women show violence as being through words, subtext and silences, rather than fists or swords" (Keatley 1994: XXXIII). Por lo tanto la cuestión sería si, al hacer referencia a Keatley y, sobre todo, a la última Churchill, se está hablando de un feminismo radical como el que se dio a lo largo de las décadas de los 60 y 70, o de un feminismo de la diferencia y de la ciudadanía "que incluya a las mujeres como mujeres en un contexto de igualdad civil y ciudadanía activa" (Pateman, en Mouffe 1999: 116).

Lo anterior viene a formular, más que a contestar, otra inquietud de quien escribe estas páginas. En su libro El retorno de lo político, Chantal Mouffe explica y comenta las tesis de algunas autoras que, como sucede con Carole Pateman, afirman la existencia de una "esencia que corresponde a las mujeres como mujeres" (Pateman, en Mouffe 1999: 116) en oposición a la de los hombres. Según Mouffe, para Pateman el ejercicio de la ciudadanía, en su más amplio sentido, es una categoría patriarcal que hay que desterrar desde el feminismo radical creando una concepción sexualmente diferenciada de dicha noción (la de ciudadanía). Partiendo de esta afirmación, será interesante cuestionar hasta qué punto se está sosteniendo, cuando leemos el teatro de Keatley y, sobre todo, en el caso de Churchill, que los postulados sobre la especificidad de "la condición femenina se basa[rían] en la identificación de las mujeres como mujeres con la maternidad" (Mouffe 1999: 116). Como lectora de estas obras, surge la pregunta por la visión del derecho y el ejercicio de la ciudadanía por parte de los personajes femeninos de Churchill y de Keatley, o, para ser más exactos, la pregunta acerca de si son algunas, o muchas, o todas sus conductas forjadoras de ciudadanía, más allá de su identificación con la capacidad y la experiencia de la maternidad. Si nos centramos en el sentido más filosófico de esta noción tendremos que abrir el espacio teórico a autores como Fernando Bárcena, quien ha dejado dicho que "la idea de la ciudadanía, más que la mera adquisición de un 'estatus' —un conjunto de derechos reconocidos por un Estado- es una práctica de compromiso" (Bárcena 1995: 279). Como el ejercicio de la ciudadanía implica la práctica de un compromiso, es sensato apuntar que dicho compromiso no se ejercerá si no existe previamente una conciencia moral cívica que posibilite el ejercicio del juicio político. Esta dimensión ética de lo cívico entendida como un conjunto de prácticas que los individuos ejercitan de manera consciente e inconsciente por decisión propia y por la influencia que ejerce sobre ellos el medio familiar y cultural es la que se expone a través de personajes doloridos, desarraigados, violentos a veces, en el teatro de las dos autoras británicas. Ahora bien, la noción diferenciadora de Pateman, seguida también por Sara Ruddick y Jean Bethke Elshtain (Mouffe 1999), choca frontalmente con la que sostienen Mary Díetz y la propia Mouffe. Estas autoras afirman, al contrario de lo que sostienen Pateman, Ruddick y Bethke, que la idea que vincula la práctica social democrática con la maternidad no puede ser convincentemente sostenida puesto que la maternidad es una actividad no política, distintiva y especial (Mouffe 1999: 114). Es decir, la noción y el ejercicio de la ciudadanía sólo pueden llevarse a cabo desde la mirada y una acción "colectiva, inclusiva y generalizada" (Mouffe 1999: 115). Mouffe cree en una ciudadanía en la que la diferencia sexual sea considerada como no pertinente, lo que conduciría a una normalización de las relaciones sociales desde el punto de vista de la diferencia sexual. Ello no significaría "la total desaparición de la diferencia sexual como distinción pertinente” (Mouffe 1999: 118) sino que más bien implicaría el compromiso de hombres y mujeres por un ejercicio distinto 
en lo que respecta a qué es ser ciudadano y miembro de una comunidad democrática. En todo caso, si entendemos, con Mouffe, que la democracia verdaderamente radical y plural se desarrolla desde "la afirmación de la libertad y la igualdad para todos" (Mouffe 1999: 120), es necesario abogar por un grado de compromiso de los individuos en las distintas instancias de la vida. Estoy hablando, pues, de un compromiso ético y político radical. A este respecto, hay que decir que tanto Churchill como Keatley, lo mismo que otras plumas dramáticas coetáneas, han prestado atención a la voz de los que no tienen voz, referido ello, sobre todo, a las mujeres que no tienen voz o cuya palabra ha sido desoída. A lo anterior se une otra característica fundamental de este teatro, tal es el modo en el que se articulan los estados internos de los personajes y del clima político del tiempo en el que se desarrollan los trasuntos argumentales de las obras. Mediante una técnica teatral depurada y transgresora, estas autoras logran que el público se cuestione qué es en realidad lo que quiere enfatizar su teatro. Por ejemplo, es recurso frecuente, sobre todo en las obras de Churchill escritas en las décadas de los 70 y 80, el que una actriz tome el papel de un hombre o viceversa. Así se hace referencia constante a las diferencias de género. Además, mediante este procedimiento las autoras enfrentan al público con una técnica que enfatiza que el actor no es el personaje, produciéndose así un vacío entre las palabras y la imagen que vemos en el escenario.

Igualmente, y siguiendo con los recursos técnicos utilizados por Churchill y Keatley, las dos autoras muestran el paso del tiempo como un factor que posibilita no sólo la revisión de la historia personal sino también la colectiva, pero que además extiende las posibilidades de reflexión sobre la vida a través de las distintas generaciones. Esto es claro en el caso de Top Girls y, sobre todo, en el planteamiento y desarrollo de My Mother Said I Never Should. En las dos obras, los personajes parecen estar buscando continuamente un motivo que les haga pensar que sus vidas están llenas de sentido. Baste recordar, por ejemplo, el momento en el que, en Top Girls, Marlene se pregunta por qué ella y las otras mujeres que le rodean están siempre tan tristes (Churchill 1982: 72). Por su parte, Griselda, con su actitud resignada, representa a la mujer que cree que quizá exista una parcela de felicidad destinada a ella en algún lugar, aunque en realidad no se da cuenta de que sufre aceptando pasivamente su dolor mediante una conducta conformista y dócil porque lo considera una experiencia natural, destinada a ser vivida por todas las mujeres. Como se aprecia a lo largo de la obra, los personajes están dispuestos a intercambiar experiencias entre ellos porque se ha puesto énfasis en el valor de la comunicación entre los distintos miembros del grupo, en este caso del grupo de amigas, pero resulta inevitable preguntarse si actuando de esta manera están evaluándose a sí mismos al mismo tiempo.

Como es evidente, no se pueden emitir juicios o valoraciones críticas sobre estas obras si no se tiene en cuenta la respuesta del lector o del público que acude a la representación teatral y el momento histórico en el que se produjeron estas obras. Por lo que se refiere a Top Girls, el texto fue escrito después de que Churchill realizara una visita a los Estados Unidos a principios de los años 80 , donde tuvo la oportunidad de escuchar a un grupo de estudiantes que opinaban sobre el ejemplo positivo que suponía Margaret Thatcher para las mujeres británicas, especialmente para las más jóvenes, así como las políticas seguidas por su gobierno en los años en los que era Primera Ministra. El comentario sirvió como revulsivo a Churchill y así Top Girls se convirtió en una de las obras más conocidas y aclamadas de la dramaturga. Con el empleo de la técnica conocida como "overlapping 
dialogues" o diálogos coincidentes, montadas las frases de los personajes que dialogan unas sobre otras, Churchill desarrolló un sistema novedoso en la semiótica teatral. Esta técnica que, según Price, Churchill habría heredado de Harold Pinter, (1999), dota de gran naturalidad a los diálogos y actúa como una "disruptive consciousness in which meanings are revealed in a new way" (Sullivan, en Price 1999: 6). Esta concepción nueva del orden establecido, actualizada por la vía de la expresión lingüística, ha sido valorada por la crítica como uno de los mayores logros de Churchill (Tong, en Price 1999: 6).

En lo tocante a My Mother Said I Never Should, existe en el desarrollo de la obra un claro interés por establecer los puntos de contacto y las diferencias entre las distintas generaciones que representan las protagonistas. Con el empleo de una técnica estructural que recuerda el planteamiento formal de Top Girls, en esta obra puede observarse una temática que une la literatura teatral de Keatley con la de Churchill, como las relaciones entre madres e hijas, el papel que ejerce la familia como el medio social idóneo donde se desarrollan los lazos humanos más profundos y los conflictos más inflamables, el papel de la educación y de la cultura, del trabajo y de la promoción en la vida laboral frente al interés del sujeto por la búsqueda de la libertad individual, y la combinación de todos estos factores en el papel asignado a las mujeres a lo largo de las distintas generaciones a lo largo del siglo XX. ¿Cuáles son las razones por las que Churchill y Keatley hacen coincidir a estos personajes mezclando edades y generaciones? En mi opinión, y atendiendo a la manera en la que se articula el recurso de la técnica de los overlapping dialogues en las dos autoras, se puede hablar del interés por ofrecer una muestra oral del abismo que existe entre los discursos de los géneros y entre las generaciones, entre las culturas oriental y occidental. Asimismo, las autoras indagarían en la necesidad de buscar medios para lograr un estadio diferente entre el nivel de cualidades comunicativas del lenguaje — un lenguaje propio de cada comunidad - $\mathrm{y}$, al mismo tiempo, un lenguaje radicalmente humano, útil para toda la humanidad. Lo anterior quedaría enmarcado en la importancia que se otorga al valor de preservar el papel de la memoria no sólo individual sino colectiva.

De manera similar a lo que ocurre en las obras de Churchill, en la de Keatley apenas hay lugar para los hombres. Al interrogante que indaga sobre el lugar que ocupan los hombres en estas obras dramáticas y al papel que se les ha reservado no se puede responder sino que la visión que se ofrece del mundo masculino es negativa. Los hombres aportan a la vida de gran parte de estas mujeres una carga de obstáculos, reales o simbólicos, que están dirigidos o influyen, de alguna manera, en estas protagonistas, por lo que el matiz que desprende su presencia en las obras es desolador y adusto. Ocurre así con Jack y con Pope, con el amante de Nijo, con los jefes de Jackie y Marlene en Top Girls, o con las tres protagonistas mayores — no así en el caso de la joven Rosie - de My Mother Said I Never Should.

La crudeza y la desolación presentes en estas producciones hacen obligada la mención de su componente pesimista y permiten que nos preguntemos si, a tenor de lo que se puede leer en ellas, existe un poco de luz para el futuro. Queda expuesta, sin embargo, la necesidad de mantener el coraje para seguir soñando.

Así, en My Mother Said I Never Should, Rosie representa a la mujer joven cuya historia no es paralela a la de su madre o su abuela aunque en ocasiones contiene similares elementos biográficos. Con Rosie se ofrece una imagen muy positiva de la juventud, siendo éste el único personaje mediante el que se aprecia la visión de la existencia hacia el 
horizonte de la esperanza: "-DORIS: At least you're not wounded inside" (Keatley 1994: 90). Es evidente, además, que la oportunidad de la joven para ejercer el derecho a la ciudadanía viene dado por la evolución de la situación política y social del tiempo que le ha tocado vivir. Su conducta y su vida permiten pensar que es posible vivir en una sociedad democrática en la que el derecho a y el ejercicio de la ciudadanía no serán íntegramente pervertidos (Keatley 1994: 53, 81- 84, 89).

El efecto que van produciendo en el público la suma de todos los elementos señalados y las técnicas utilizadas no es otro que la crítica y la toma de conciencia de la realidad que otros han vivido y viven. El tú se convierte, pues, en un factor imprescindible mediante el que se integran el pasado colectivo y el individual y por medio del cual se logra que el sufrimiento y la violencia no sean meros recursos textuales para crear un efecto meramente visual. El compromiso, pues, estriba en lograr el desarrollo de un principio intelectual y artístico que posibilite el paso desde la dificultad que ofrecen la intervención individual de cada personaje y la necesidad de respetar el silencio escénico que da voz a todos los personajes - en las últimas creaciones de Churchill sobre todo- presentado como una técnica escénica en la que todos hablan, hasta la necesidad de desenmascarar la influencia que ejercen las ideologías en la construcción de la identidad y en el modo en el que el sujeto femenino se percibe a sí mismo. Por lo tanto, una de las conclusiones que se desprende de esta práctica que ambas autoras ponen al servicio del drama es que no siempre es posible llegar a un verdadero entendimiento mediante el lenguaje. De esta manera, la voz dramática se carga de sentido político. Utilizándolo tal y como lo hacen Churchill y Keatley, por medio de multitud de técnicas y referencias intertextuales, se fuerzan las posibilidades del lenguaje hasta el extremo, potenciándose así el énfasis en el uso de otros lenguajes, como la danza, la luz, los colores, o las barreras que impone el uso deliberado de la lengua de manera enfrentada a la que se espera en lo que conocemos como los principios de la comunicación - por ejemplo, explotando el uso de la onomatopeya, reduciendo, no en sentido peyorativo, la lengua al sonido y jugando con ese recurso.

\section{REFERENCIAS BIBLIOGRÁFICAS}

ASTON, E., An Introduction to Feminism and Theatre, London, Routledge, 1995.

ASTON, E. / REINELT, J., The Cambridge Companion to Modern British Women Playwrights, Cambridge, Cambridge University Press, 2000.

BERENGUER, A./PÉREZ, M., Tendencias del Teatro Español durante la Transición Política (1975-1982), Madrid, Biblioteca Nueva, 1998.

BÁRCENA ORBE, F. (1995), "La educación moral de la ciudadanía. Una filosofía de la educación cívica", Revista de Educación, 307, 1995 pp. 275- 308.

CHURCHILL, C., Top Girls, London, Methuen, 1982.

CHURCHILL, C., The Skriker, London, Nick Hern Books, 1993.

KEATLEY, C., My Mother Said I Never Should, London, Methuen, [1988], 1994.

KLEIN-HAGEN, H/LÓPEZ ROMÁN, B., Teorías feministas y sus aplicaciones al teatro feminista británico contemporáneo, Granada, Comares, 2001.

MOUFFE, C., El retorno de lo político, Barcelona, Paidós [1993], 1999. 
PRADO PÉREZ, J. R., Revisiones críticas del teatro alternativo británico: 1968- 1990, Castelló, Universitat Jaume I, 2000.

PRICE, J. A., "The Language of Caryl Churchill: the Rhythms of Feminist Theory, Acting Theory and Gender Politics". Internet. 15- 04- 2005. < http://www.women writers.net>.

WERTEMBAKER, T., Our Country's Good, London, Methuen, [1988], 1995.

WU, D., Making Plays. Interviews with Contemporary British Dramatists, 2000 and Directors, New York, St. Martin's Press, 2000. 